\title{
Flocking in Stationary and Non-stationary Environments: A Novel Communication Strategy for Heading Alignment
}

\author{
Eliseo Ferrante, Ali Emre Turgut, Nithin Mathews, \\ Mauro Birattari, and Marco Dorigo
}

IRIDIA, CoDE, Université Libre de Bruxelles, Brussels, Belgium

\begin{abstract}
We propose a novel communication strategy inspired by explicit signaling mechanisms seen in vertebrates, in order to improve performance of self-organized flocking for a swarm of mobile robots. The communication strategy is used to make the robots match each other's headings. The task of the robots is to coordinately move towards a common goal direction, which might stay fixed or change over time.

We perform simulation-based experiments in which we evaluate the accuracy of flocking with respect to a given goal direction. In our settings, only some of the robots are informed about the goal direction. Experiments are conducted in stationary and non-stationary environments. In the stationary environment, the goal direction and the informed robots do not change during the experiment. In the non-stationary environment, the goal direction and the informed robots are changed over time. In both environments, the proposed strategy scales well with respect to the swarm size and is robust with respect to noise.
\end{abstract}

\section{Introduction}

In nature, we observe different activities performed by animals living in groups. Such activities require collective decision-making even when few individuals have the needed information. This information then spreads in the group according to different mechanisms, depending on the species. In some insect and fish species, information is transferred implicitly without any signaling mechanism [1|2]. Conversely, other species utilize explicit signaling mechanisms such as vocalization. For example, mountain gorillas switch between some daily activities (resting to travelling/feeding) very rapidly using vocalization [3. In a honeybee swarm, when scouts agree on a new nest site, they fly rapidly towards the nest while signaling the right direction to the rest of the swarm [4].

Coordinated motion of animals is an example of activities which require collective decision-making. For example, flocks of birds and schools of fish move and maneuver coherently as if they were a super-organism [5]. Recent work in biology showed that a group of animals can be guided to particular locations (food sources) even if only a minority of the group is aware of the location [1. 
The first studies of flocking in swarm robotics were inspired by Reynolds' seminal work [6], one of the first regarding flocking outside biology. Reynolds obtained a realistic computer animation of a flock of birds through three simple concurrent behaviors: separation (avoiding collisions), cohesion (staying close to neighbors) and alignment (heading in the same direction as neighbors). These behaviors are all based on local sensing and local decision rules. Separation and cohesion control, denoted in the rest of the paper as proximal control, is the aggregate behavior controlling the relative distance between individuals.

In Reynolds' work, in order to perform alignment, each individual is assumed to sense the velocity of its neighbors without noise. This assumption is unrealistic in robotics. Subsequent works have tried to relax this assumption. These works can be organized into three different categories. The first category does not feature an explicit alignment behavior. Instead, it tries to obtain alignment intrinsically via other behaviors such as homing [7, leader-following [8] and light-following [9]. The second category achieves alignment by resorting to the emulation of a heading sensor [10] or to the estimation of the heading of neighbors [1]. In the third and last category, heading information is spread within the swarm via local communication [12]. Turgut et al. [13] proposed an algorithm belonging to the third category. A robot measures its heading with respect to the North using a compass and broadcasts it periodically so that the heading is sensed "virtually" by its neighbors. With this method, Turgut et al. [13] achieved self-organized flocking in a random direction.

Çelikkanat et al. 14, inspired by the implicit decision-making mechanisms of some animal species [15/2], extended the flocking behavior proposed by Turgut et al. [13] by providing a goal direction to some of the robots ("informed" robots). They observed that a large swarm can be guided by only a few robots, which is in accordance with theoretical results [1].

In this paper, inspired by both biological [34] and swarm robotics ideas [14], we propose a new method for heading alignment. The proposed strategy that we call information-aware communication, uses only local communication, which means that robots can communicate only with their neighbors within a given range and in their line of sight. Our system is composed of informed and uninformed robots: informed robots relay the goal direction and uninformed ones just send the average of the messages they receive from their neighbors. We compare the novel information-aware communication with heading communication, where all of the robots always send the measured heading information to their neighbors by still relying on local communication only. The latter was used by Turgut et al. and Çelikkanat et al. [1314].

We present the results of two sets of experiments. One is conducted in a stationary environment, in which the goal direction and the informed indivuals do not change during the experiment. The other is conducted in a non-stationary environment, in which both the goal direction and the informed robots are changed at regular time intervals during the experiment. 


\section{Methodology}

The methodology we use to design the flocking behavior is based on artificial physics [9]. At each control step, a virtual force vector is computed as:

$$
\mathbf{f}=\alpha \mathbf{p}+\beta \mathbf{h}+\gamma \mathbf{g}
$$

where $\mathbf{p}$ is the proximal control vector; $\mathbf{h}$ is the heading alignment vector; $\mathbf{g}$ is the vector that indicates the goal direction. The vectors $\mathbf{p}$ and $\mathbf{h}$ are each calculated by a behavior (explained in Section 2.1 and Section 2.2 respectively). The goal direction vector $\mathbf{g}$ is available to some robots, whereas for the others $\mathbf{g}=\mathbf{0}$. The weights $\alpha, \beta$ and $\gamma$ define the relative contribution of the different force components. In this paper, we do not tune these parameters for obtaining optimal performance, but we set them to $\alpha=1, \beta=5$ and $\gamma=10$ to reflect our prior knowledge on the relative importance of the three components.

\subsection{Proximal Control Behavior}

The proximal control behavior assumes that a robot perceives the relative position (range and bearing) of its neighbors in close proximity. This is realized using LEDs and an omni-directional camera as in 12. Let $k$ denote the number of robots perceived at a given time, $d_{i}$ and $\phi_{i}$ denote the range and bearing measurements of the $i^{\text {th }}$ robot, respectively. The virtual force $\mathbf{p}$ is given by:

$$
\mathbf{p}=\sum_{i=1}^{k} p_{i} e^{j \phi_{i}},
$$

where $p_{i} e^{j \phi_{i}}$ are vectors expressed in polar coordinates. $p_{i}$ is calculated as a function of $d_{i}$ using a force function $p_{i}\left(d_{i}\right)$ as in [16]. $p_{i}$ is repulsive when $d_{i}$ is smaller than the desired distance $(D)$ and it is attractive when $d_{i}$ is greater than $D$. The function is:

$$
p\left(d_{i}\right)=-\frac{2 D^{2}}{d_{i}^{3}}+\frac{2}{d_{i}},
$$

\subsection{Heading Alignment Behavior}

The heading alignment behavior assumes that, using an onboard light sensor, a robot $r$ measures its heading $\left(\theta_{r}\right)$ with respect to the common reference frame represented by a light source. The robot receives an angle $\theta_{i}$ from its $i^{\text {th }}$ neighbor. The value sent by each neighbor depends on which communication strategy is used, as explained in the following. Each received angle is transformed into robot $r$ body-fixed reference frame 1 . Having received $k$ angles from its $k$ neighbors,

\footnotetext{
${ }^{1}$ We define two reference frames. One is the reference frame common to all of the robots, and the other is the body-fixed reference frame specific to each robot. The body-fixed reference frame is fixed to the center of a robot: its $x$-axis is coincident with the rotation axis of the wheels and its $y$-axis points to the front of the robot.
} 
robot $r$ calculates the average heading vector as:

$$
\mathbf{h}=\frac{\sum_{i=1}^{k} e^{j \theta_{i}}}{\left\|\sum_{i=1}^{k} e^{j \theta_{i}}\right\|},
$$

where $\|\cdot\|$ denotes the norm of a vector.

The proposed communication strategy, that we call information-aware communication strategy, is explained in the following.

Information-aware communication: This communication strategy assumes that robots are aware of whether they are provided with the goal direction $\mathbf{g}$ or not, that is whether they are informed or non-informed. This awareness mechanism is implemented by measuring the length of the goal direction vector $\mathbf{g}$ : the robot considers itself non-informed if $\mathbf{g}=\mathbf{0}$ and informed if $\mathbf{g} \neq \mathbf{0}$. Each robot then communicates the following information: if it is non-informed, it sends $\angle \mathbf{h}(\angle \cdot$ denotes the angle of a vector $)$ to its immediate neighbors; otherwise, it sends $\angle \mathbf{g}$. The intuition behind this strategy is the following: if the robot is non-informed, it should facilitate the diffusion of the information originating from the informed robots; if it is informed, it should then directly propagate the information about the goal location to its immediate neighbors. The information then eventually reaches the entire swarm thanks to the uninformed robots. Note that the awareness of each robot is only used to determine which information should be sent, and is never directly communicated to neighboring robots (i.e., each robot never knows if a message is received from an informed or a noninformed robot).

As a baseline comparison, we implemented another communication strategy that we call heading communication strategy. This strategy is similar to the one used in 13 .

Heading communication: This communication strategy consists in the local communication of the robot's own current heading $\theta$ measured with respect to the common reference frame. In the original work [13], this strategy was used to simulate robots that are able to measure the heading of their neighbors when the actual measurement is not physically possible.

\subsection{Motion Control}

The computed virtual force vector $\mathbf{f}$ is mapped into rotational speed of the wheels. First, using Newton's second law of motion, the target velocity $\mathbf{u}_{\text {target }}$ is computed:

$$
\mathbf{u}_{\text {target }}=\mathbf{u}^{t}+\frac{\mathbf{f} \Delta t}{m},
$$

where $\Delta t$ is the control-step size, $m$ is the mass of the robot and $\mathbf{u}^{t}$ is the current velocity of the robot. The target velocity $\mathbf{u}_{\text {target }}$ cannot be followed directly by the robot due to its non-holonomic constraints. Thus, it is mapped into the robot's forward velocity $\mathbf{u}^{t+1}$, which points in the direction of the $y-$ axis and has magnitude $u=\left\|\mathbf{u}^{t+1}\right\|$ set to: 


$$
u= \begin{cases}\left(\frac{\mathbf{u}_{\text {target }}}{\left\|\mathbf{u}_{\text {target }}\right\|} \cdot \frac{\mathbf{u}^{t}}{\left\|\mathbf{u}^{t}\right\|}\right) u_{\max }, & \text { if } \mathbf{u}_{\text {target }} \cdot \mathbf{u}^{t} \geq 0 ; \\ 0, & \text { otherwise. }\end{cases}
$$

where $u_{\max }$ is set to $0.036 \mathrm{~m} / \mathrm{s}$.

The angular velocity $\omega$ of the robot is determined by a proportional controller that calculates the deviation of the desired angle from the current heading of the robot: $\omega=K_{p}\left(L_{\text {target }}-\angle \mathbf{u}^{t}\right)$, where $K_{p}$ is a proportionality constant whose value is set to $0.5 s^{-1}$. Finally, the rotation speeds of the left $\left(N_{L}\right)$ and right $\left(N_{R}\right)$ motors are set to:

$$
N_{L}=\left(u+\frac{\omega}{2} l\right) \frac{1}{r} ; N_{R}=\left(u-\frac{\omega}{2} l\right) \frac{1}{r},
$$

where $l$ is the distance between the wheels and $r$ is their radius.

\section{Experiments}

In this section, we first introduce the metrics and the experimental setup used to evaluate the proposed methodology. We then present the results in a stationary and a non-stationary environment.

\subsection{Metrics}

In flocking, we are interested in having a group of robots that move compactly, coherently, within their sensory range and without collisions. Furthermore, the group should be aligned towards a common direction (in our case the goal direction) and move towards that direction. In this paper, we use two metrics as in 14]: order and accuracy.

Order: The order metric $\psi$ is used to measure the angular order of the robots. $\psi \approx 1$ when the group has a common heading and $\psi \ll 1$ when each robot is pointing in a random, different direction. The order is defined as:

$$
\psi=\frac{1}{N}\|\overline{\mathbf{a}}\|=\frac{1}{N}\left\|\sum_{i=1}^{N} e^{j \theta_{i}}\right\|,
$$

where $N$ is the total number of robots in the experiment, and $\overline{\mathbf{a}}$ is the vectorial sum of the measured headings of the $N$ robots.

Accuracy: The accuracy metric $\delta$ is used to measure how accurately close to the target direction (dependent on the task) robots are moving. $\delta \approx 1$ when robots are perfectly aligned (which corresponds also to a high value for the order metric $\psi \approx 1$ ) towards the correct direction of motion. As in 1 , accuracy can be defined as:

$$
\delta=1-\frac{\sqrt{2(1-\psi \cos (\angle \overline{\mathbf{a}}-\angle \mathbf{g}))}}{2}
$$

where $\angle \overline{\mathbf{a}}$ is the direction of $\overline{\mathbf{a}}$ and $\angle \mathbf{g}$ is the goal direction with respect to the common reference frame. 


\subsection{The Task and the Experimental Setup}

In our experiments, $N$ mobile robots are placed at random positions and with random orientations in an empty arena of 5 meters $\times 5$ meters. Each robot is a realistic simulation of a foot-bot, in development for the Swarmanoid project 2 . We utilized the following sensors and actuators: i) A light sensor, that is able to perceive a noisy light gradient around the robot. It is used to measure $\theta_{r}$, the orientation of robot $r$ with respect to a common light source. ii) A range and bearing communication system, with which a robot can send a message to other robots that are within 2 meters and in line of sight [17. iii) Two wheels actuators, that are used to control independently the left and right wheels speed of the robot. iv) 24 LEDs and a camera, which are used to detect distance and bearing from other robots in the proximal control behavior (see Section 2.1). We conducted two sets of experiments.

Stationary environment: In a stationary environment, a proportion $\rho$ of randomly selected robots are given the information about the goal direction $\mathbf{g}$. All the other robots remain uninformed for the entire duration of the simulation. In every run, we randomly choose $\mathbf{g}$, as well as the selection of robots that are informed. The duration of one run is 100 simulated seconds.

Non-stationary environment: A non-stationary environment consists of four stationary phases of equal length. The proportion of informed robots $\rho$ is kept fixed during the entire run. However, at the beginning of every stationary phase, the informed robots are reselected at random. Also, the goal direction $\mathrm{g}$ changes randomly from one stationary phase to the next one. The duration of one run is 250 simulated seconds.

In the stationary environment, we study the effect of changing the swarm size $N$ and the proportion of informed robots $\rho$. In the non-stationary environment, we also study the effect of noise in the heading alignment vector $\mathbf{h}$. We modeled this noise as a uniformly distributed random variable controlled by a scaling parameter $\sigma \in[0,1]$, which is used to add noise to $\theta_{r}$, the robot's measured heading: $\tilde{\theta_{r}}=\theta_{r}+\mathcal{U}(-\sigma 2 \pi,+\sigma 2 \pi)$. For each experimental setting, we execute 100 runs and we report the average results.

\subsection{Results in Stationary Environments}

The effect of varying the swarm size $N$ is shown in Figure 1a, The informationaware communication strategy outperforms the classical heading communication strategy, in the sense that it achieves higher accuracy both with small (10) and large (100) swarms. In both strategies, the convergence speed is higher with the smaller swarm size. This can be explained by the fact that smaller swarms have smaller inertia than larger swarms. However, in the heading communication strategy, the accuracy level reached with a larger swarm is higher than the one

2 http://www. swarmanoid.org 


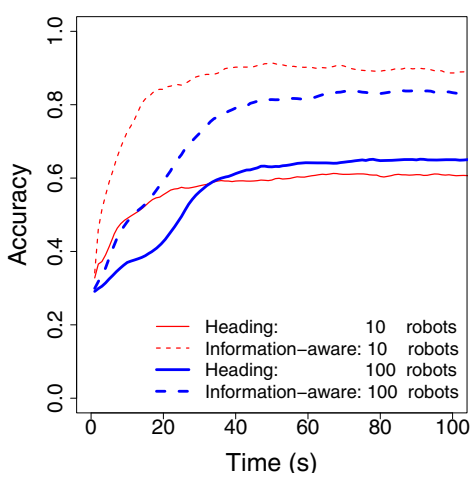

(a)

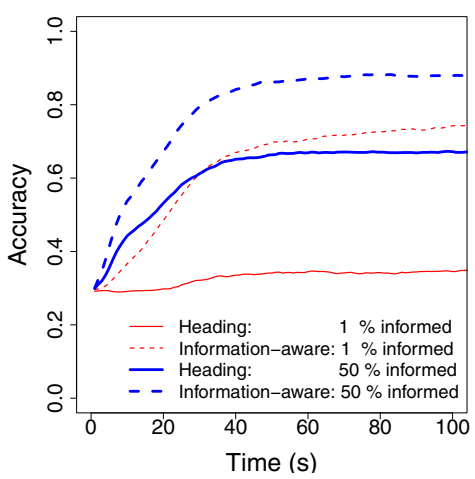

(b)

Fig. 1. Information-aware communication against heading communication in a stationary environment:(a) effect of varying swarm size $N$ with fixed $\rho=0.25$ and $\sigma=0$; (b) effect of varying the percentage of informed robots $\rho$ with fixed $N=100$ and $\sigma=0$

achieved with a smaller swarm. This result is consistent with the findings of [1, which state that the needed proportion of informed robots to achieve a given level of accuracy becomes smaller for increasing swarm sizes.

The effect of varying the proportion of informed robots $\rho$ is shown in Figure 1b, Also in this case, the information-aware communication strategy outperforms the classical heading communication. In both strategies, more informed robots corresponds to higher accuracy. With a proportion of $1 \%$ informed robots (corresponding to 1 robot), the classical alignment communication strategy cannot achieve an increasing accuracy over time, which means that at the end of the simulation robots are randomly oriented as they were at the beginning. Differently, the proposed information-aware communication strategy can cope also with a very small proportion of informed robots.

\subsection{Results in Non-stationary Environments}

The effect of varying the swarm size $N$ is shown in Figure 2a, The same trends observed in the stationary environment are also present here: The informationaware communication strategy always outperforms the classical alignment communication. However, here we observe an interesting phenomenon: The accuracy convergence speed of the information-aware communication strategy in the initial stationary phase is lower than the one in the subsequent three stationary phases. This can be explained by the order shown in Figure 2b, The initial order of the system is very low, and gets higher and higher towards the end of the first stationary phase. When the change in the environment occurs, order decreases but does not reach the initial, very low values for any of the techniques. This means that the swarm is able to make a transition from an ordered state to another ordered state without a detrimental impact on the order itself, which in turn corresponds to faster adaptation to changes in the environment. 


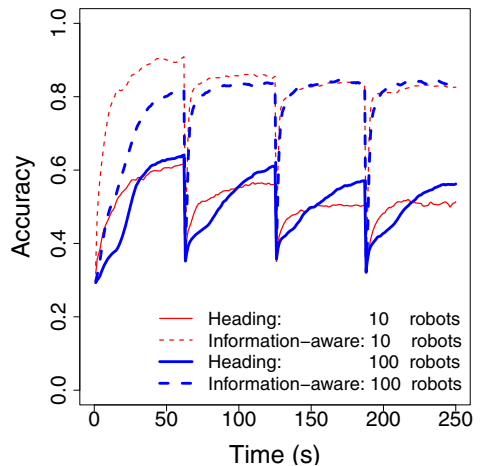

(a)

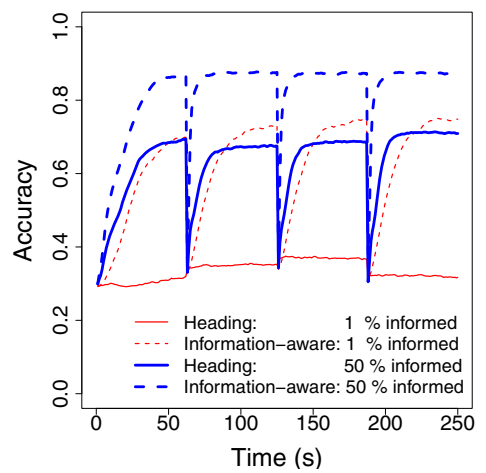

(c)

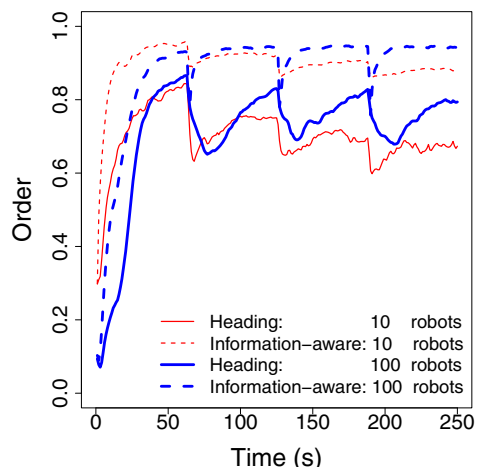

(b)

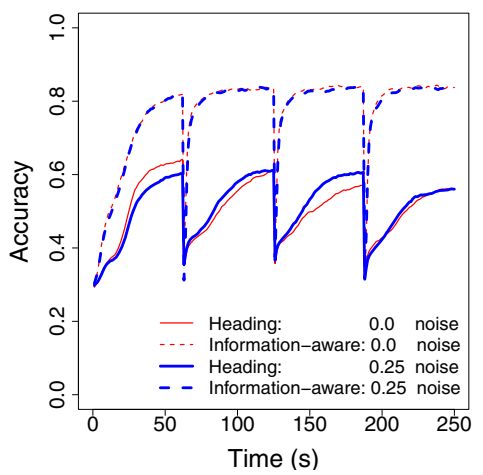

(d)

Fig. 2. Information-aware communication againts heading communication in a nonstationary environment: effect on the accuracy (a) and on the order (b) of varying swarm size $N$ with fixed $\rho=0.25$ and $\sigma=0$, (c) effect of varying the percentage of informed robots $\rho$ with fixed $N=100$ and $\sigma=0$; (d) effect of adding noise $\sigma=0.25$ with fixed $N=100$ and $\rho=0.25$.

The effect of varying the proportion of informed robots $\rho$ is shown in Figure 2c The same trends observed in the stationary case apply in this case. Hence, the information-aware communication strategy scales well to the non-stationary case even when only $1 \%$ of the robots (in this case only one robot) are informed.

Finally, the effect of adding noise $\sigma$ in the alignment is shown in Figure 2d. As we can see, noise has a non-significant impact on the accuracy of the informationaware communication strategy, which still continues to outperform the classical alignment communication strategy.

\section{Conclusions and Future Work}

In this paper, we proposed a communication strategy, called information-aware communication, for heading alignment in self-organized flocking. In the system, 
a swarm of robots is decomposed into informed and uninformed robots: informed robots possess information about a goal direction, whereas uninformed robots do not. The proposed strategy works as follows: each robot communicates the goal direction when it is informed, whereas it communicates the average of its neighbors messages when it is uninformed. We compared this strategy with the heading communication strategy, similar to those used in the literature, in which each robot communicates directly its own heading.

We conducted two sets of experiments. The first set is executed in a stationary environment, where the goal direction and the informed robots do not change over time. The second set is executed in a non-stationary environment, where both the goal direction and the informed robots change over time. Results show that the proposed information-aware communication strategy always outperforms the heading communication strategy. Furthermore, the proposed approach is also robust against noise in the alignment, and it achieves high accuracy values even if only few robots in a large swarm are informed.

The presented work can be extended in many ways. First, a more complete scenario may include multiple sources of different information which can be conflicting, and perceived by different robots in different ways. In this scenario, we may need the swarm to be coherent while heading towards a common direction, or split to different sub-groups, depending on the application. To cope with this scenario, we might need to include some measure of information quality, perceived by each robot, which can be used to determine a new communication strategy. Secondly, we plan to port the presented work into real robots. We believe that the set of assumptions made in this work are all compatible with future real robots experiments, with the exception of the one in which we assume that all robots are able to perceive a common environmental cue. As a matter of fact, the foot-bot robots that we will use are not equipped with a compass, and the light sensor cannot be used for such an aim because the perception of light is not uniform in the swarm due to the robots shadowing each other or to their varying distance from the light source. To avoid the need of a common environmental cue, we plan to extend the information-aware communication strategy to incorporate the capabilities of a situated communication mechanism, in which also the range and bearing of the sender is available and has a meaning.

Acknowledgments. This work was supported by the $S W A R M A N O I D$ project funded by the Future and Emerging Technologies programme (IST-FET) of the European Commission (grant IST-022888). M. Birattari and M. Dorigo acknowledge support from the F.R.S.-FNRS of the French Community of Belgium.

\section{References}

1. Couzin, I.D., Krause, J., Franks, N.R., Levin, S.A.: Effective leadership and decision-making in animal groups on the move. Nature 433, 513-516 (2005)

2. King, A.J., Johnson, D.D.P., Van Vugt, M.: The origins and evolution of leadership. Current biology 19(19), 911-916 (2009) 
3. Stewart, K.J., Harcourt, A.H.: Gorillas' vocalizations during rest periods: signals of impending departure? Behaviour 130(1), 29-40 (1994)

4. Beekman, M., Fathke, R., Seeley, T.: How does an informed minority of scouts guide a honeybee swarm as it flies to its new home? Animal Behaviour 71(1), 161-171 (2006)

5. Camazine, S., Franks, N.R., Sneyd, J., Bonabeau, E., Deneubourg, J.L., Theraulaz, G.: Self-Organization in Biological Systems. Princeton University Press, Princeton (2001)

6. Reynolds, C.: Flocks, herds and schools: A distributed behavioral model. In: Stone, M.C. (ed.) SIGGRAPH 1987: Proc. of the 14th Annual Conference on Computer graphics and Interactive Techniques, pp. 25-34. ACM, New York (1987)

7. Matarić, M.J.: Interaction and Intelligent Behavior. PhD thesis, MIT, MA (1994)

8. Kelly, I., Keating, D.: Flocking by the fusion of sonar and active infrared sensors on physical autonomous robots. In: Proc. of the Third Int. Conf. on Mechatronics and Machine Vision in Practice, pp. 14-17 (1996)

9. Spears, W.M., Spears, D.F., Hamann, J.C., Heil, R.: Distributed, physiscs-based control of swarms of vehicles. Autonomous Robots 17, 137-162 (2004)

10. Holland, O., Woods, J., Nardi, R., Clark, A.: Beyond swarm intelligence: the ultraswarm. In: Proc. of the IEEE Swarm Intelligence Symposium, Piscataway, NJ, pp. 217-224. IEEE Press, Los Alamitos (2005)

11. Hayes, A., Dormiani-Tabatabaei, P.: Self-organized flocking with agent failure: Offline optimization and demonstration with real robots. In: Proc. of the IEEE Int. Conf. on Robotics and Automation, Piscataway, NJ, pp. 3900-3905. IEEE Press, Los Alamitos (2002)

12. Campo, A., Nouyan, S., Birattari, M., Groß, R., Dorigo, M.: Negotiation of goal direction for cooperative transport. In: Dorigo, M., Gambardella, L.M., Birattari, M., Martinoli, A., Poli, R., Stützle, T. (eds.) ANTS 2006. LNCS, vol. 4150, pp. 191-202. Springer, Heidelberg (2006)

13. Turgut, A.E., Çelikkanat, H., Gökçe, F., Şahin, E.: Self-organized flocking in mobile robot swarms. Swarm Intelligence 2(2), 97-120 (2008)

14. Çelikkanat, H., Turgut, A.E., Şahin, E.: Guiding a robot flock via informed robots. In: Asama, Kurokawa, Ota, Sekiyama (eds.) Distributed Autonomous Robotic Systems (DARS 2008), Berlin, Germany, pp. 215-225. Springer, Heidelberg (2008)

15. Reebs, S.G.: Can a minority of informed leaders determine the foraging movements of a fish shoal? Animal behaviour 59, 403-409 (2000)

16. Tanner, H.G., Jadbabaie, A., Pappas, G.J.: Stable flocking of mobile agents part II: Dynamic topology. In: Proc. of the 42nd IEEE Conference on Decision and Control, Piscataway, NJ, vol. 2, pp. 2016-2021. IEEE Press, Los Alamitos (2003)

17. Roberts, J., Stirling, T., Zufferey, J., Floreano, D.: 2.5d infrared range and bearing system for collective robotics. In: Papanikolopoulos, N., Sugano, S., Chiaverini, S., Meng, M. (eds.) IEEE/RSJ International Conference on Intelligent Robots and Systems, Piscataway, NJ. IEEE Press, Los Alamitos (2009) 